\title{
Optimizing Management of Patients With Barrett's Esophagus and Low-Grade or No Dysplasia Based on Comparative Modeling
}

Amir-Houshang Omidvari, ${ }^{*}$ Ayman Ali, ${ }^{\ddagger, \S}$ William D. Hazelton, " Sonja Kroep, Minyi Lee, ${ }^{\ddagger}$ Steffie K. Naber, ${ }^{*}$ Brianna N. Lauren," Sassan Ostvar," Ellen Richmond, ${ }^{\#}$ Chun Yin Kong, ${ }^{\ddagger}$ Joel H. Rubenstein, ${ }^{* *, \neq \neq}$ Iris Lansdorp-Vogelaar, ${ }^{*}$ Georg Luebeck," Chin Hur, ${ }^{\ddagger, \uparrow}$ and John Inadomi ${ }^{\S}$

${ }^{*}$ Department of Public Health, Erasmus MC University Medical Center Rotterdam, Rotterdam, The Netherlands; ${ }^{\ddagger}$ Institute for Technology Assessment, Massachusetts General Hospital, Boston, Massachusetts; ${ }^{\S}$ Tulane University School of Medicine, New Orleans, Louisiana; "Program in Computational Biology, Fred Hutchinson Cancer Research Center, Seattle, Washington; \#Division of Cancer Prevention, National Cancer Institute, Rockville, Maryland; **Veterans Affairs Center for Clinical Management Research, Ann Arbor, Michigan; ${ }^{\ddagger \ddagger}$ Barrett's Esophagus Program, Division of Gastroenterology, University of Michigan, Ann Arbor, Michigan; "Irving Medical Center, Columbia University, New York, New York; ${ }^{\S}$ Division of Gastroenterology, Department of Medicine, University of Washington, Seattle, Washington

BACKGROUND \& AIMS:

Endoscopic treatment is recommended for patients with Barrett's esophagus (BE) with high-grade dysplasia, yet clinical management recommendations are inconsistent for patients with BE without dysplasia (NDBE) or with low-grade dysplasia (LGD). We used a comparative modeling analysis to identify optimal management strategies for these patients.

METHODS:

We used 3 independent population-based models to simulate cohorts of 60-year-old individuals with BE in the United States. We followed up each cohort until death without surveillance and treatment (natural disease progression), compared with 78 different strategies of management for patients with NDBE or LGD. We determined the optimal strategy using cost-effectiveness analyses, at a willingness-to-pay threshold of $\$ 100,000$ per qualityadjusted life-year (QALY).

RESULTS:

In the 3 models, the average cumulative incidence of esophageal adenocarcinoma was 111 cases, with costs totaling \$5.7 million per 1000 men with BE. Surveillance and treatment of men with BE prevented $23 \%$ to $75 \%$ of cases of esophageal adenocarcinoma, but increased costs to $\$ 6.2$ to $\$ 17.3$ million per 1000 men with BE. The optimal strategy was surveillance every 3 years for men with NDBE and treatment of LGD after confirmation by repeat endoscopy (incremental cost-effectiveness ratio, $\$ 53,044 / Q A L Y$ ). The average results for women were consistent with the results for men for LGD management, but the optimal surveillance interval for women with NDBE was 5 years (incremental cost-effectiveness ratio, $\$ 36,045 / Q A L Y$ ).

CONCLUSIONS: $\quad$ Based on analyses from 3 population-based models, the optimal management strategy for patient with BE and LGD is endoscopic eradication, but only after LGD is confirmed by a repeat endoscopy. The optimal strategy for patients with NDBE is endoscopic surveillance, using a 3-year interval for men and a 5-year interval for women.

Keywords: ICER; EET; Radiofrequency Ablation; RFA; EAC.

Abbreviations used in this paper: BE, Barrett's esophagus; EAC, esophageal adenocarcinoma; EACMo, Esophageal Adeno Carcinoma Model; EET, endoscopic eradication therapy; HGD, high-grade dysplasia; LGD, low-grade dysplasia; MISCAN, Microsimulation Screening Analysis; MSCE, Multistage Clonal Expansion; NDBE, nondysplastic Barrett's esophagus; QALY, quality-adjusted life-year; RFA, radiofrequency ablation.
Most current article

(C) 2020 by the AGA Institute $1542-3565 / \$ 36.00$

https://doi.org/10.1016/j.cgh.2019.11.058 


\section{See editorial on page 1930.}

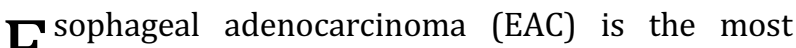
E common subtype of esophageal cancer in the United States and other Western countries. ${ }^{1}$ The incidence and mortality of EAC have increased dramatically since the $1970 \mathrm{~s}^{2}$ Barrett's esophagus (BE) is the only known precursor lesion for EAC. ${ }^{3}$ Therefore, clinical guidelines recommend endoscopic surveillance and/or treatment of $\mathrm{BE}$ patients depending on the presence and grade of dysplasia. There is general consensus among gastrointestinal professional societies regarding management of BE patients with high-grade dysplasia (HGD): endoscopic eradication therapy (EET) using endoscopic mucosal resection or endoscopic submucosal dissection to remove endoscopically visible lesions, followed by radiofrequency ablation (RFA) of flat dysplastic and metaplastic epithelium should be performed. However, there is uncertainty about the optimal surveillance interval for nondysplastic BE patients (NDBE) and whether EET or surveillance is the optimal management strategy for patients with low-grade dysplasia (LGD) (Appendix Table 1).

Because of interobserver variation in the diagnosis of dysplasia, particularly LGD, most clinical guidelines recommend that a histologic diagnosis of LGD should be confirmed by a second pathologist with expertise in gastroenterological pathology. The American College of Gastroenterology guidelines recommend endoscopic eradication therapy for patients with confirmed LGD, although endoscopic surveillance every 12 months is an acceptable alternative. ${ }^{4}$ Of note, the American Gastroenterological Association recommends a repeat endoscopy after proton pump inhibitor therapy for 8 to 12 weeks to reduce inflammatory and regenerative changes that could be misdiagnosed as dysplasia, before initiating EET. ${ }^{5}$

Optimal BE management should be guided by the relative benefits and harms of competing strategies. Because clinical trials are of insufficient duration to assess cancer mortality and survival accurately, simulation modeling studies are helpful to compare overall effectiveness and cost effectiveness of different $\mathrm{BE}$ management strategies throughout the lifetime of individual patients and across populations. Population models use aggregates of hypothetical individual event histories associated with key components of a disease process, based on available relevant data, to estimate population-level effects of interventions on outcomes and the comparative effectiveness of a variety of interventions. ${ }^{6}$ A number of previous modeling studies, including those conducted by our group, reported inconsistent results on the management of LGD and NDBE patients. ${ }^{7-11}$ Although these studies examined the influence of variations in model parameters on conclusions, the influence of variations in model structural assumptions (eg, regression of dysplasia or metaplasia) on conclusions could not be examined because most studies were based on individual models. Moreover, prior

\section{What You Need to Know}

\section{Background}

Management recommendations for patients with Barrett's esophagus without dysplasia or with lowgrade dysplasia (LGD) are inconsistent.

\section{Findings}

Based on analyses from 3 population-based models, the optimal management strategy for patient with Barrett's esophagus and LGD is endoscopic eradication, but only after LGD is confirmed by a repeat endoscopy. The optimal strategy for patients with Barrett's esophagus without dysplasia is endoscopic surveillance, using a 3-year interval for men and a 5year interval for women.

\section{Implications for patient care}

These findings have implications for management of patients with Barrett's esophagus and can inform policy development and practice decisions.

studies were not calibrated adequately to current population-based EAC incidence and mortality. Finally, the impact of repeat endoscopy to confirm LGD after high-dose acid suppression to reduce false-positive test results was not assessed. ${ }^{7-9}$

In this study we conducted a comparative effectiveness analysis using 3 independently developed models to determine the most cost-effective management strategy for BE patients with LGD and the optimal interval for surveillance of patients with NDBE.

\section{Methods}

We used 3 independent population-based models that are part of the National Cancer Institute Cancer Intervention and Surveillance Modeling Network to compare the benefits, harms, and costs of different management strategies for BE patients.

\section{Cancer Intervention and Surveillance Modeling Network Esophageal Adenocarcinoma Models}

Three models were used for this analysis, as follows: (1) Microsimulation Screening Analysis (MISCAN)-EAC model from Erasmus University Medical Center (Rotterdam, The Netherlands) and the University of Washington (Seattle, WA); (2) Esophageal Adeno Carcinoma Model (EACMo), originally developed at the Massachusetts General Hospital (Boston, MA) and currently at the Columbia University Medical Center (New York, NY); and (3) Multistage Clonal Expansion for EAC (MSCE-EAC) model from the Fred Hutchinson Cancer Research Center (Seattle, WA) (see the Appendix for details). Full descriptions of the models are published and available online. $^{12}$ 


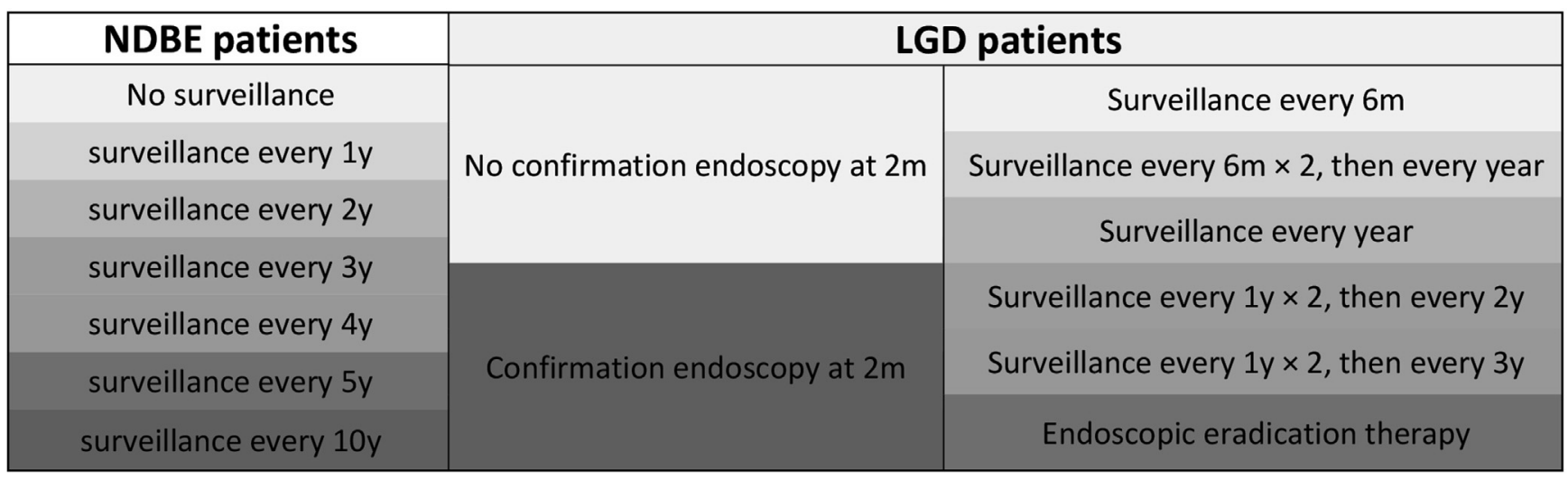

Figure 1. Differences in simulated strategies. The 78 simulated strategies were combinations of different options presented in each column of this figure. LGD, low-grade dysplasia; NDBE, nondysplastic Barrett's esophagus.

\section{Population Simulated}

For the main analysis, a hypothetical cohort of US white men diagnosed with BE at age 60 in 2010 was simulated and followed up until death or age 100, whichever occurred first, without surveillance and BE treatment (natural history). We separately simulated a cohort of US white women diagnosed with $\mathrm{BE}$ at age 60 who underwent the same strategies.

\section{Strategies Assessed}

We compared 78 different BE management strategies: the large number results from permutations in surveillance intervals and differences in management of LGD. In all strategies, HGD patients received EET consisting of endoscopic mucosal resection of visible lesions followed by RFA. The strategies varied in LGD management, as follows: surveillance with different intervals, or EET, with or without confirmation of LGD by a repeat endoscopy after 2 months of high-dose acid suppression; and in NDBE management, as follows: no surveillance or surveillance with different intervals $(1,2,3,4,5$, and 10 y) (see Figure 1 and Appendix Table 2 for details).

\section{Surveillance and Treatment Assumptions}

Assumptions about the performance of surveillance endoscopy were based on the current literature. We assumed that individuals without BE could be misdiagnosed as NDBE with a probability of 0.075 (falsepositive rate) whereas NDBE patients were missed with a probability of 0.125 (false-negative rate). ${ }^{13}$ Other patients were detected but could be misdiagnosed as NDBE, LGD, HGD, or EAC with the probabilities specified in Appendix Table 3. Misdiagnosis can be the result of sampling error, misclassification, or disease regression. Only 1 model (MSCE-EAC model) explicitly simulated all 3 pathways. Two models (MISCAN-EAC and EACMo) captured all misdiagnoses in misclassification and sampling errors, but did not distinguish these pathways separately. In the MISCAN-EAC model, additional misdiagnosis may have occurred because of disease regression. However, the contribution to total misdiagnosis was negligible.

All BE patients who received EET entered posttreatment surveillance depending on the initial dysplasia status of the patient and the outcome of the EET (Appendix Table 4). ${ }^{5,14}$ Assumptions concerning the efficacy of endoscopic therapy are presented in Appendix Table 5 based on the pretreatment dysplasia status of the BE patients. ${ }^{15}$ For recurrence of dysplasia or metaplasia after initial EET, patients received RFA touch-ups followed by surveillance (Appendix Table 4). Patients could undergo a maximum of 3 RFA touch-ups, after which management was limited to surveillance for early diagnosis of cancer. ${ }^{7}$ The stop age of surveillance and treatments was assumed to be 80 years.

The duration of initial EET was assumed to be 2 years in all strategies. ${ }^{7}$ Fifty-five percent of patients underwent endoscopic mucosal resection of mucosal irregularities (nodules, masses, or ulcers) before RFA. ${ }^{16}$ On average, patients received 3.55 sessions of RFA during the initial EET period. ${ }^{7}$ Complication rates of EET and endoscopies were estimated based on the literature (see common model inputs in Appendix Table 5).

\section{Cost and Utility}

The costs of surveillance endoscopy and EETs were based on Centers for Medicare and Medicaid Services reimbursement rates. ${ }^{17}$ Costs and utilities of cancer care, perforation resulting from endoscopy, and complications resulting from stricture were estimated based on the published literature (Appendix Table 5). All costs were adjusted for year 2015 using the US consumer price index. $^{18}$

\section{Outcomes}

For each strategy, we computed health outcomes including the incidence of symptomatic and surveillance- 
Table 1. Results of Base-Case Natural History and Selected BE Management Strategies (Average of the Models) per 1000 BE Male Patients

\begin{tabular}{|c|c|c|c|c|c|c|}
\hline $\begin{array}{l}\text { Strategy and } \\
\text { outcome }\end{array}$ & Natural history & ND 5 y LGD $1 y^{2}$ & ND 3 y LGD $1 y^{a}$ & ND 3 y LGD $1 y^{b}$ & ND 3 y LGD EET ${ }^{b}$ & ND 3 y LGD EET ${ }^{a}$ \\
\hline EAC incidence & 111 & 59 & 52 & 51 & 32 & 38 \\
\hline EAC mortality & 77 & 26 & 21 & 21 & 12 & 15 \\
\hline Endoscopies & - & 7408 & 9882 & 10,234 & 9968 & 9158 \\
\hline Initial EET & _- & 187 & 216 & 220 & 635 & 358 \\
\hline RFA touch-ups & - & 84 & 100 & 104 & 337 & 178 \\
\hline \multicolumn{7}{|l|}{ Cost, $\$ 1000$} \\
\hline Cancer care & 5668 & 3709 & 3302 & 3240 & 2162 & 2477 \\
\hline Endoscopies & - & 4149 & 5593 & 5830 & 5864 & 5327 \\
\hline $\begin{array}{l}\text { EET/touch- } \\
\text { ups }\end{array}$ & - & 994 & 1146 & 1178 & 3635 & 1994 \\
\hline Total cost & 5668 & 8851 & 10,041 & 10,247 & 11,662 & 9799 \\
\hline LY & 14,566 & 14,854 & 14,878 & 14,881 & 14,923 & 14,909 \\
\hline QALY & 14,523 & 14,825 & 14,850 & 14,853 & 14,888 & 14,881 \\
\hline
\end{tabular}

BE, Barrett's esophagus; EAC, esophageal adenocarcinoma; EET, endoscopic eradication therapy; LGD, low-grade dysplasia; LY, life-years; ND, nondysplastic Barrett's esophagus; QALY, quality-adjusted life-year; RFA, radiofrequency ablation.

${ }^{a}$ LGD is not confirmed by a repeat endoscopy at 2 months.

${ }^{b}$ LGD is confirmed by a repeat endoscopy at 2 months.

detected EAC, EAC mortality, complications of endoscopies and treatments, and life-years and qualityadjusted life-years (QALYs) per 1000 BE patients. In addition, we calculated the number of endoscopies, EETs, RFA touch-ups, complications, and cancer care years to estimate the total costs of surveillance and treatment per strategy. The outcomes were analyzed for each model separately, then the average results of the 3 models were presented per strategy as the base case. ${ }^{19}$ Individual model results were presented in the sensitivity analysis.

\section{Analysis}

The optimal strategy was identified through an incremental cost-effectiveness analysis from a third-party payer perspective using the average results of the 3 models. ${ }^{19}$ Costs and QALYs were discounted at an annual rate of $3 \%$. We performed an incremental cost-effective analysis using a willingness-to-pay threshold (WTP) of $\$ 100,000 /$ QALY to determine the optimal BE management strategy, which has been suggested to be consistent with societal willingness to pay for medical interventions. $^{20}$

\section{Sensitivity Analyses}

We performed 7 sensitivity analyses to assess the robustness of our results to our structural and parameter assumptions. First, we considered the results of each model separately. In addition, we conducted a sensitivity analysis using a more intensive post-treatment surveillance of LGD patients consistent with the American College of Gastroenterology recommendations, ${ }^{4}$ with endoscopic surveillance every 6 months in the first year after EET and then annually in the strategies in which LGD patients underwent EET. We also conducted 1-way sensitivity analyses applying higher and lower values than the main analysis for EET efficacy and recurrence rates after complete eradication of intestinal metaplasia after EET (Appendix Table 6). Finally, we performed a probabilistic sensitivity analysis in which cost and utility values, as well as the rate of complications of endoscopy and EET, were varied simultaneously (Appendix Table 7). In the probabilistic sensitivity analysis, we also considered lower $(\$ 50,000)$ and higher $(\$ 150,000)$ thresholds for cost effectiveness.

\section{Results}

\section{Results for Men}

Without surveillance or EET, the models predicted an average EAC cumulative incidence and mortality of 111 cases and 77 deaths per 1000 BE patients, respectively, with a total cost of $\$ 5.7$ million for the care of incident EAC cases (Table 1). Surveillance or EET prevented $23 \%$ to $75 \%$ of EAC cases and decreased mortality by $31 \%$ to $88 \%$ while increasing costs to $\$ 6.2$ to $\$ 17.3$ million, depending on the management strategy (Appendix Table 8).

The average results of selected different BE management strategies are presented in Table 1 to compare the effect of different BE management strategies. Surveillance with a 5-year interval for NDBE and a 1-year interval for LGD patients without a confirmation endoscopy decreased EAC incidence to 59 cases $(-47 \%)$ and EAC mortality to $26(-66 \%)$ per $1000 \mathrm{BE}$ patients. The total costs increased to $\$ 8.9$ million while gaining 302 more QALYs compared with the natural history. Reducing the surveillance interval for NDBE patients to 3 years prevented more EAC cases and achieved greater QALYs, but the total costs increased (Table 1). 
Table 2. The Results of a Cost-Effectiveness Analysis per 1000 BE Male and 1000 Female Patients

\begin{tabular}{|c|c|c|c|}
\hline Strategy & Cost, $\$ 1000$ & QALY & ICER, \$/QALY ${ }^{a}$ \\
\hline \multicolumn{4}{|l|}{ Per 1000 BE male patients } \\
\hline Natural history & 5668 & 14,523 & - \\
\hline ND 0 LGD EET ${ }^{b}$ & 6176 & 14,728 & 2476 \\
\hline ND 5 y LGD EET ${ }^{b}$ & 8672 & 14,855 & 19,779 \\
\hline ND 4 y LGD EET ${ }^{b}$ & 9148 & 14,869 & 32,850 \\
\hline ND 3 y LGD EET ${ }^{b, d}$ & 9799 & 14,881 & 53,044 \\
\hline ND 2 y LGD EET ${ }^{b}$ & 11,518 & 14,892 & 156,313 \\
\hline ND 2 y LGD EET ${ }^{c}$ & 13,253 & 14,894 & $1,105,045$ \\
\hline ND 1 y LGD EET ${ }^{b}$ & 15,698 & 14,896 & $1,446,520$ \\
\hline \multicolumn{4}{|l|}{ Per 1000 BE female patients } \\
\hline Natural history & 3534 & 16,268 & \\
\hline ND 0 LGD $1 \mathrm{y} \times 2,3 \mathrm{y}^{\mathrm{C}}$ & 4450 & 16,405 & 6716 \\
\hline ND 0 LGD EET ${ }^{b}$ & 4553 & 16,419 & 7561 \\
\hline ND 5 y LGD EET ${ }^{b, e}$ & 7448 & 16,499 & 36,045 \\
\hline ND 4 y LGD EET ${ }^{b}$ & 8064 & 16,504 & 118,233 \\
\hline ND 3 y LGD EET ${ }^{b}$ & 8839 & 16,508 & 202,874 \\
\hline ND 3 y LGD EET ${ }^{c}$ & 10,869 & 16,511 & 700,093 \\
\hline
\end{tabular}

BE, Barrett's esophagus; EET, endoscopic eradication therapy; ICER, incremental cost-effectiveness ratio; LGD, low-grade dysplasia; ND, nondysplastic Barrett's esophagus; QALY, quality-adjusted life-year; $1 \mathrm{y} \times 2$, $3 \mathrm{y}$, surveillance every 1 year for 2 years, thereafter every 3 years.

${ }^{a}$ The ICERs were calculated before rounding the numbers reported for costs and QALYs in this table.

${ }^{b} \mathrm{LGD}$ is confirmed by a repeat endoscopy at 2 months.

${ }^{c}$ LGD is not confirmed by a repeat endoscopy at 2 months.

${ }^{d}$ The optimal strategy was EET after confirmation by repeat endoscopy for LGD patients and surveillance every 3 years for NDBE patients. Strategies that were dominated are not shown in the table.

${ }^{\text {e}}$ The optimal strategy was EET after confirmation by repeat endoscopy for LGD patients and surveillance every 5 years for NDBE patients. Strategies that were dominated are not shown in the table.

EET for patients with LGD increased QALYs and costs, and decreased EAC incidence and mortality compared with surveillance for LGD. The strategy with EET for LGD patients after endoscopic confirmation with surveillance every 3 years for NDBE patients decreased EAC incidence and mortality to 38 cases $(-66 \%)$ and 15 deaths $(-81 \%)$ per $1000 \mathrm{BE}$ patients compared with natural history, respectively. The costs increased to $\$ 9.8$ million while gaining 358 more QALYs than natural history. EET for LGD without endoscopic confirmation resulted in slightly higher QALYs compared with EET for LGD with confirmation, but the costs increased by almost $\$ 2$ million per $1000 \mathrm{BE}$ patients (Table 1).

\section{Cost-Effectiveness Analysis}

Strategies using EET for LGD patients (either with or without confirmation endoscopy) were generally more effective than strategies with surveillance for LGD patients (Appendix Table 8). In men, only strategies limiting EET to patients confirmed to have LGD on endoscopic confirmation after acid suppression were cost effective (Table 2, Figure 2A). Considering a WTP threshold of $\$ 100,000$, the optimal BE management strategy for men was EET for endoscopically confirmed
LGD after 2 months of acid suppression, and surveillance every 3 years for NDBE patients (incremental costeffectiveness ratio, $\$ 53,044 / \mathrm{QALY}$ ).

\section{Results for Women}

Predicted EAC cases in women were lower than in men (75 and 111 per 1000 in women and men, respectively). Consequently, QALYs gained from BE surveillance and treatment were lower in women than in men, and incremental costs per QALY gained for similar BE management strategies were higher. Most cost-effective strategies in women included EET for LGD confirmed by endoscopy after 2 months of acid suppression, similar to results for men (Table 2, Figure 2B). Because of the higher incremental costs per QALY gained in women, the optimal strategy was surveillance every 5 years for NDBE (incremental cost-effectiveness ratio, $\$ 36,045$ / QALY).

\section{Sensitivity Analysis}

All 3 models consistently found EET to be the optimal strategy for male BE patients with LGD (Table 3). However, because costs per QALY gained were generally lower in the MISCAN-EAC model, the optimal strategy for male NDBE patients varied from surveillance every 2 years (MISCAN-EAC) to surveillance every 3 years (EACMO and MSCE-EAC) (Appendix Tables 9-14).

The optimal strategy for men did not change in the 1-way sensitivity analyses: applying a more intensive surveillance strategy after treatment of LGD patients, a higher or lower value for EET efficacy, or a higher or lower value for recurrence rate after complete eradiation of metaplasia after receiving EET (Table 3, Appendix Tables 15-19). Results also were robust to wide variations in assumptions on complication rates, cost, and utility values. The optimal BE management strategy for men (EET after confirmation by repeat endoscopy after acid suppression for LGD patients and surveillance every 3 years for NDBE patients) was optimal in $100 \%$ of runs in probabilistic sensitivity analyses for WTP thresholds of $\$ 67,500$ to $\$ 137,500 /$ QALY (Figure 3).

\section{Discussion}

Our comparative modeling analysis indicated that the most cost-effective strategy for patients with BE and LGD is EET, but only if the LGD is confirmed by a repeat endoscopy after 2 months of high-dose, acid-suppression therapy. Our analysis also found that the most costeffective strategy for NDBE patients is surveillance every 3 years in men but every 5 years in women.

We found that none of the strategies using surveillance for LGD patients, or EET without confirmation of LGD, were cost effective in men. For women, only 1 of 7 efficient strategies used surveillance for LGD. This 


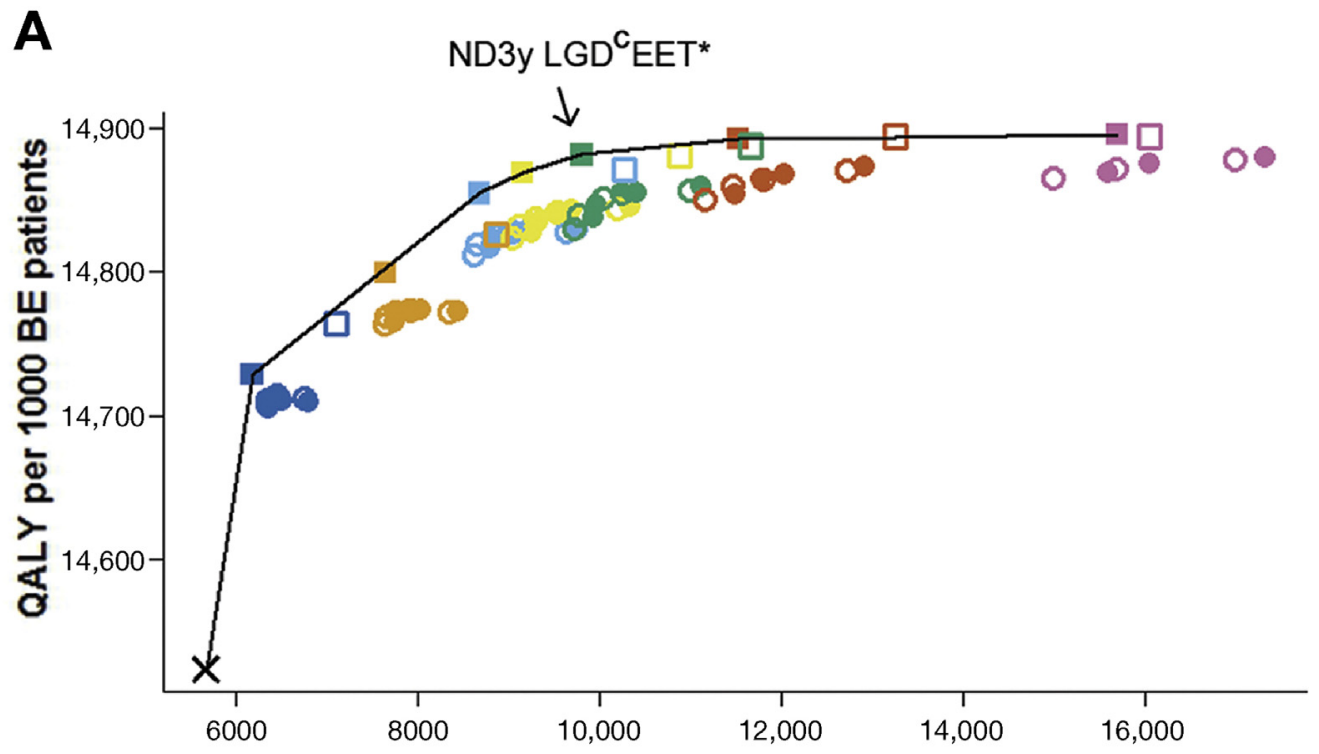

\section{COST per $1000 \mathrm{BE}$ patients (US $\$ 1,000$ )}

B

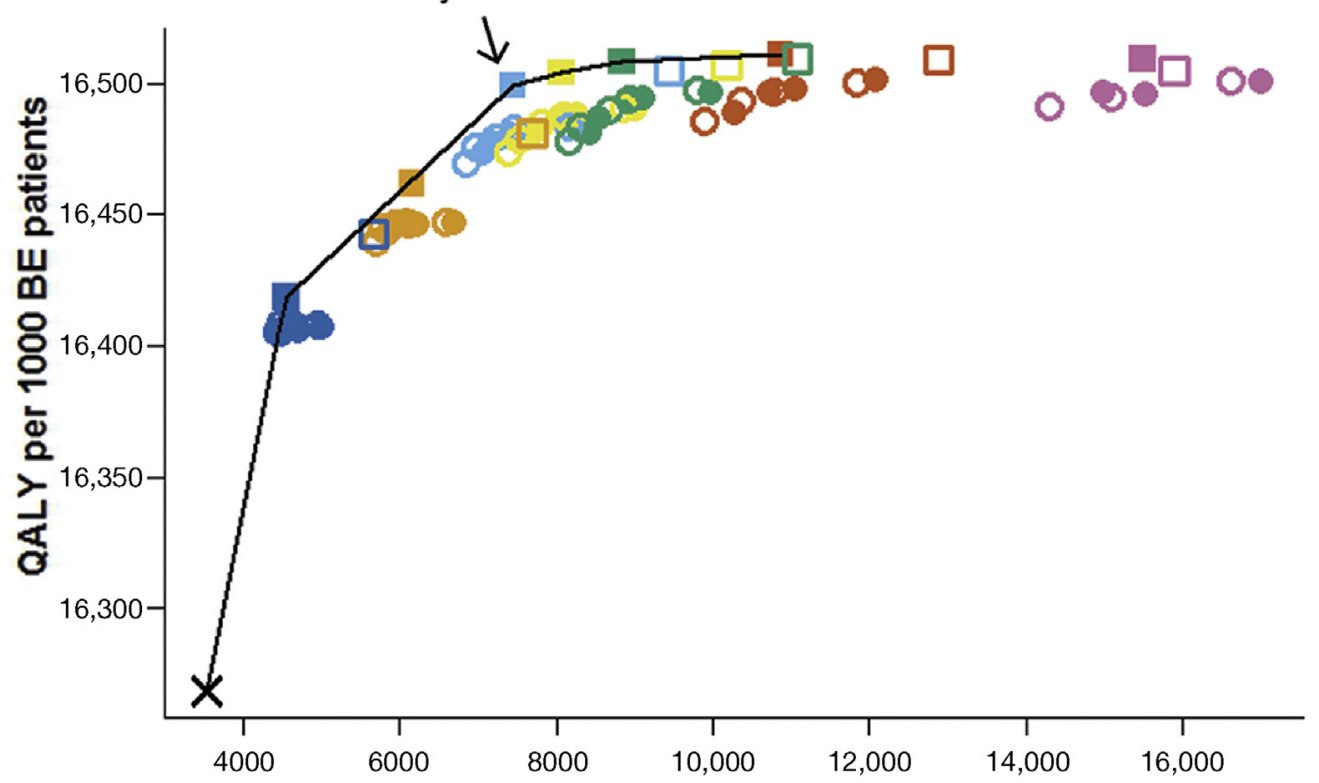

COST per 1000 BE patients (US $\$ 1,000$ )

NDBE surveillance

\section{No surveillance \\ Every 10 years \\ Every 5 years}

Every 4 years

\section{Every 3 years \\ Every 2 years}

Every 1 year

\section{LGD management}

EET for LGD with confirmation

$\square$ EET for LGD without confirmation

LGD surveillance with confirmation

LGD surveillance without
Figure 2. Results of a costeffectiveness analysis for $(A)$ men and $(B)$ women. ${ }^{\mathrm{C} C o n f i r m e d}$ by a repeat endoscopy at 2 months. ${ }^{*}$ The optimal strategy for men was EET after confirmation by repeat endoscopy for LGD patients and surveillance every 3 years for nondysplastic Barrett's esophagus patients with an incremental costeffectiveness ratio of \$53,044/QALY gained, and the optimal strategy for women was EET after confirmation by repeat endoscopy for LGD patients and surveillance every 5 years for nondysplastic Barrett's esophagus patients with an incremental costeffectiveness ratio of \$36,045/QALY gained, assuming a willingness-topay threshold of $\$ 100,000 /$ QALY. BE, Barrett's esophagus; EET, endoscopic eradication therapy; LGD, low-grade dysplasia; ND, nondysplastic Barrett's esophagus; QALY, quality-adjusted life-year. finding was consistent between the individual models and the wide variation in assumptions examined in the sensitivity analyses. Despite the potential harms and cost of endoscopic therapy, EET of LGD reduces the number of endoscopies required for surveillance of LGD patients because of prolonged surveillance intervals after successful treatment and generally prevents more EAC cases than strategies using only surveillance for LGD. 
Table 3. Optimal Management Strategy by Analysis for Male BE Patients

\begin{tabular}{|c|c|c|c|c|c|c|}
\hline Analysis & $\mathrm{NDBE}^{a}$ & LGD & EAC prevented ${ }^{b}$ & Net cost $(\$ 1000)^{b}$ & QALY gained ${ }^{b}$ & ICER, \$/QALY \\
\hline Base-case $^{d}$ & $3 y$ & $2 \mathrm{mo},{ }^{e} \mathrm{EET}$ & $66 \%$ & 4131 & 358 & 53,044 \\
\hline MISCAN-EAC model & $2 y$ & $2 \mathrm{mo}^{e}$ EET & $67 \%$ & 6676 & 452 & 78,140 \\
\hline EACMo model & $3 y$ & $2 \mathrm{mo}^{e}$ EET & $67 \%$ & 3514 & 365 & 67,225 \\
\hline MSCE-EAC model & $3 y$ & $2 \mathrm{mo}^{e} \mathrm{EET}$ & $67 \%$ & 3822 & 278 & 84,564 \\
\hline More intensive post-EET surveillance ${ }^{d}$ & $3 y$ & $2 \mathrm{mo},{ }^{e}$ EET & $66 \%$ & 4785 & 353 & 68,199 \\
\hline Higher EET efficacy ${ }^{d}$ & $3 y$ & $2 \mathrm{mo}^{e} \mathrm{EET}$ & $70 \%$ & 3785 & 364 & 49,379 \\
\hline Lower EET efficacy ${ }^{d}$ & $3 y$ & $2 \mathrm{mo}^{e}$ EET & $62 \%$ & 4458 & 341 & 59,180 \\
\hline Higher recurrence rate after $\mathrm{CE}-\mathrm{IM}^{d}$ & $3 y$ & $2 \mathrm{mo}^{e}$ EET & $59 \%$ & 5007 & 338 & 66,501 \\
\hline Lower recurrence rate after CE-IM ${ }^{d}$ & $3 y$ & $2 \mathrm{mo}^{e}$ EET & $66 \%$ & 4131 & 358 & 49,115 \\
\hline
\end{tabular}

BE, Barrett's esophagus; CE-IM, complete eradication of intestinal metaplasia; EACMo, Esophageal Adeno Carcinoma Model; EET, endoscopic eradication therapy; ICER, incremental cost-effectiveness ratio; LGD, low-grade dysplasia; MISCAN, Microsimulation Screening Analysis; MSCE, Multistage Clonal Expansion; NDBE, nondysplastic Barrett's esophagus; QALY, quality-adjusted life-year.

${ }^{a}$ The numbers in the column show the optimal surveillance interval.

${ }^{b}$ Per 1000 BE patients compared with natural history.

${ }^{c}$ The ICERs were calculated before rounding the numbers reported for costs and QALYs in this table.

${ }^{d}$ Combined results of the models (mean).

${ }^{\text {e}}$ Confirmatory endoscopy at 2 months, if LGD is confirmed then an EET.

Consequently, EET strategies are not much more expensive than some surveillance-only strategies, while gaining greater QALYs. Importantly, confirmation of LGD before EET is more cost effective than EET without confirmation. Confirmatory endoscopy decreases the number of false-positive results of LGD diagnosis. Although 1 more endoscopy is required per BE patient, the additional costs are compensated by the reduced number of inappropriate EETs (ablation of misdiagnosed NDBE patients).

The 3 models are less consistent in identifying the optimal strategy for NDBE patients, although 2 models showed that surveillance every 3 years is the optimal strategy for men, the MISCAN-EAC model suggested that surveillance every 2 years is optimal. This discrepancy can be explained by different model assumptions between the 3 models (eg, on regression and progression of BE patients).

Prior analyses reported inconsistent findings regarding the management of NDBE patients. A modeling study in Australia reported that surveillance of all NDBE patients likely was not cost effective, ${ }^{21}$ whereas studies in The Netherlands and the United States reported that surveillance of NDBE patients could be cost effective

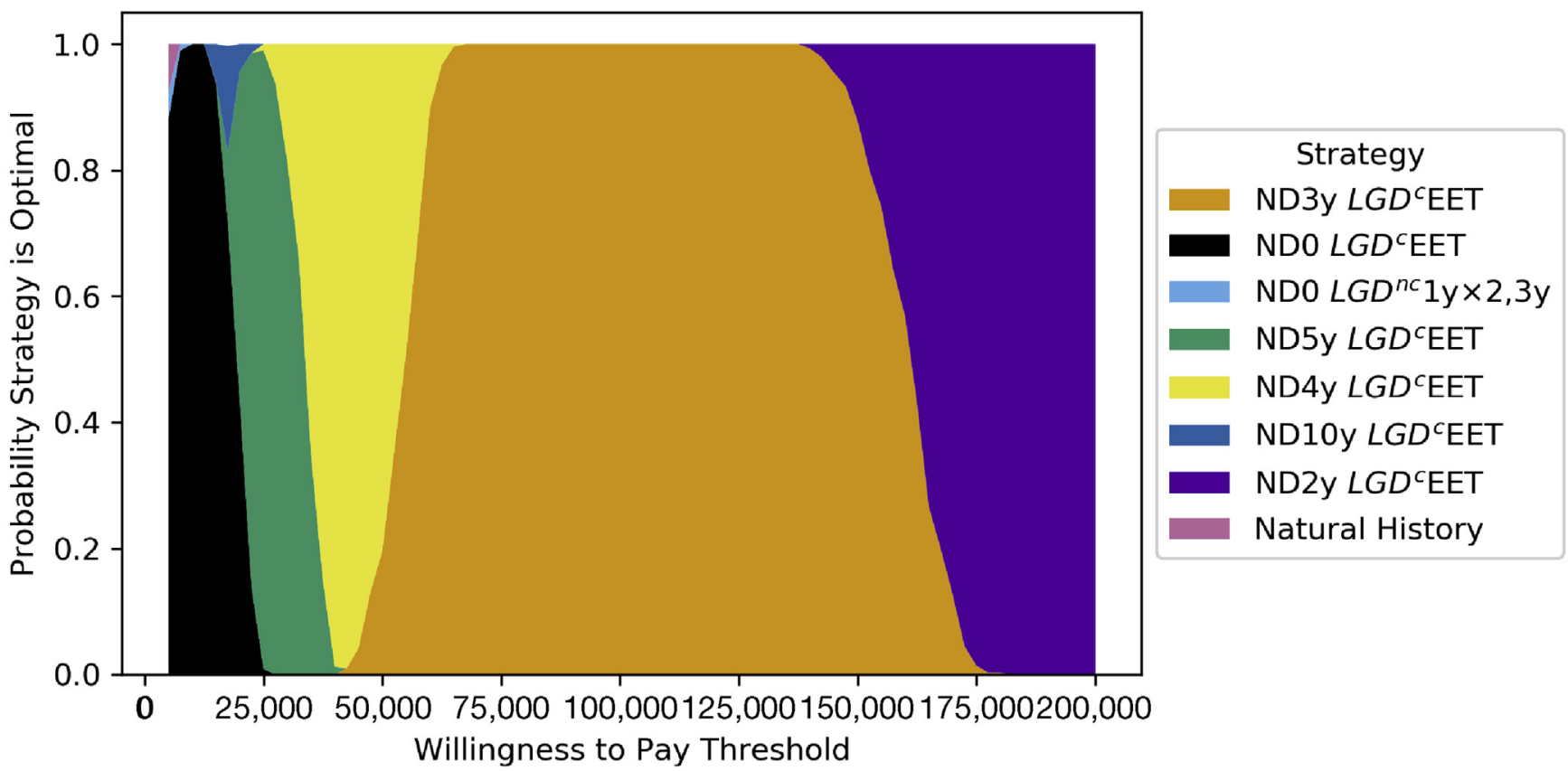

Figure 3. Results of a probabilistic sensitivity analysis by willingness-to-pay thresholds (\$ per quality-adjusted life-year) for

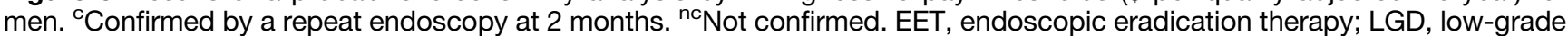
dysplasia; ND, nondysplastic Barrett's esophagus. 
using a 3- or 5-year interval, respectively. ${ }^{10,22}$ These studies were limited by the use of a single model/natural history, ${ }^{8,9}$ and they examined fewer competing management strategies.

In our analysis, we simulated cohorts of 60-year-old $\mathrm{BE}$ patients because the mean/median age of BE patients at diagnosis is older than 60 years. ${ }^{23,24}$ In all strategies, the stop age of surveillance was assumed to be 80 years. This stopping age was chosen to reflect the balance between benefits and harms of surveillance with increasing age. The US Preventive Services Task Force recommended age 75 for the stopping age of average-risk individuals for colorectal cancer and breast cancer screening. ${ }^{25,26}$ We chose the higher age of 80 because patients with BE, even NDBE, are no longer average risk.

An innovative feature of our analysis was the ability to test different structural assumptions about the natural history of progression from BE to EAC by comparing 3 independently developed models. Two models (MISCAN-EAC and MSCE-EAC) assumed that regression from HGD to LGD and from LGD to NDBE can occur in the absence of EET, while the third model (EACMo) did not allow regression to occur. The MSCE-EAC model is based on the molecular and cellular changes that underlie the progression from normal squamous epithelium to $\mathrm{BE}$ and to EAC, whereas the other 2 models are populationbased cohort simulations reflecting the clinically identifiable stages leading to EAC development. All models are calibrated independently to reproduce Surveillance, Epidemiology, and End Results data and use identical clinical parameter estimates (variable values) ${ }^{27}$; however, the manner in which these variables interact differs substantially between models. Despite these differences in model structure, our results were remarkably consistent. A further strength of our study was the ability to present a sensitivity analysis that simultaneously varied the estimated values of variables in 3 models with unique natural history assumptions about BE and EAC. For these reasons, we believe our comparative modeling results are more robust than prior studies in which analyses were limited to a single model.

Our study had several limitations. A major concern was the limited amount of published long-term EET outcome data. For this reason, short-term clinical effects were used to inform our models and extrapolated to project long-term effects, and we conducted sensitivity analyses applying higher and lower values for EET efficacy and recurrence rates after complete eradication of metaplasia. Furthermore, there was limited evidence regarding the association of multifocal LGD or LGD with nodularity and progression ${ }^{5}$; and only 1 of our models (MSCE-EAC) explicitly modeled mutations throughout the tissue that may lead to multifocal clonal growth. This is an area with less information, requiring more explorative research in the future. In addition, the underlying prevalence of $\mathrm{BE}$ driving the overall incidence of EAC is unknown and, for this reason, the models differed in the numbers of total EACs (preclinical and clinically detected) estimated over the lifetime of the cohort. Cost estimates used Centers for Medicare and Medicaid Services data, which are relevant to individuals 65 years of age and older and may have underestimated the costs incurred in individuals 60 to 64 years of age in this study. Finally, utilities were estimated from limited data and may not represent individual preferences for different health states accurately. Therefore, we conducted a probabilistic sensitivity analysis on cost and utility values to evaluate the impact of our assumptions on our results.

Notwithstanding these limitations, our analysis has important implications for the management of BE patients and can inform BE policy development and practice decisions. We focus particularly on BE patients without dysplasia or with LGD, who represent the majority of patients diagnosed with $\mathrm{BE}$. The management of $\mathrm{BE}$, particularly LGD, has received inconsistent recommendations from societies. In addition to informing US guidelines, our findings are applicable in other settings. The analyses in our study were conducted for a US cohort, but we expect similar results for BE populations in other settings with similar BE progression rates and high EAC incidence such as Northern and Western Europe. ${ }^{28-32}$

In summary, our comparative modeling analysis using 3 independent simulation models found that the most cost-effective management strategy for BE patients with LGD is EET, if LGD is confirmed by a repeat endoscopy after 2 months of high-dose, acid-suppression therapy. For NDBE patients, our analysis found the optimal cost-effective strategy is surveillance at 3-year intervals for men and at 5-year intervals for women.

\section{Supplementary Material}

Note: To access the supplementary material accompanying this article, visit the online version of Clinical Gastroenterology and Hepatology at www.cghjournal.org, and at https://doi.org/10.1016/j.cgh.2019.11.058.

\section{References}

1. Napier KJ, Scheerer M, Misra S. Esophageal cancer: a review of epidemiology, pathogenesis, staging workup and treatment modalities. World J Gastrointest Oncol 2014;6:112-120.

2. Hur C, Miller M, Kong CY, et al. Trends in esophageal adenocarcinoma incidence and mortality. Cancer 2013; 119:1149-1158.

3. Schoofs N, Bisschops R, Prenen H. Progression of Barrett's esophagus toward esophageal adenocarcinoma: an overview. Ann Gastroenterol 2017;30:1-6.

4. Shaheen NJ, Falk GW, Iyer PG, et al. ACG Clinical Guideline: diagnosis and management of Barrett's esophagus. Am J Gastroenterol 2016;111:30-50, quiz 1.

5. Wani S, Rubenstein JH, Vieth M, et al. Diagnosis and management of low-grade dysplasia in Barrett's esophagus: expert review from the Clinical Practice Updates Committee of the American Gastroenterological Association. Gastroenterology 2016;151:822-835. 
6. Rutter CM, Zaslavsky AM, Feuer EJ. Dynamic microsimulation models for health outcomes: a review. Med Decis Making 2011; 31:10-18.

7. Kroep S, Heberle CR, Curtius K, et al. Radiofrequency ablation of Barrett's esophagus reduces esophageal adenocarcinoma incidence and mortality in a comparative modeling analysis. Clin Gastroenterol Hepatol 2017;15:1471-1474.

8. Inadomi JM, Somsouk M, Madanick RD, et al. A cost-utility analysis of ablative therapy for Barrett's esophagus. Gastroenterology 2009;136:2101-2114.e1-6.

9. Hur C, Choi SE, Rubenstein JH, et al. The cost effectiveness of radiofrequency ablation for Barrett's esophagus. Gastroenterology 2012;143:567-575.

10. Das A, Wells C, Kim HJ, et al. An economic analysis of endoscopic ablative therapy for management of nondysplastic Barrett's esophagus. Endoscopy 2009;41:400-408.

11. Sonnenberg A, Soni A, Sampliner RE. Medical decision analysis of endoscopic surveillance of Barrett's oesophagus to prevent oesophageal adenocarcinoma. Aliment Pharmacol Ther 2002; 16:41-50.

12. CISNET esophagus cancer collaborators. Esophageal Cancer Model Profiles. NIH Cancer Intervention and Surveillance Modeling Network (CISNET); 2018. Available from, https://cisnet. cancer.gov/esophagus/profiles.html. Accessed June 22, 2018.

13. Provenzale D, Schmitt C, Wong JB. Barrett's esophagus: a new look at surveillance based on emerging estimates of cancer risk. Am J Gastroenterol 1999;94:2043-2053.

14. American Gastroenterological Association, Spechler SJ, Sharma P, et al. American Gastroenterological Association medical position statement on the management of Barrett's esophagus. Gastroenterology 2011;140:1084-1091.

15. Cotton CC, Wolf WA, Overholt BF, et al. Late recurrence of Barrett's esophagus after complete eradication of intestinal metaplasia is rare: final report from Ablation in Intestinal Metaplasia Containing Dysplasia Trial. Gastroenterology 2017; 153:681-688 e2.

16. Gupta M, lyer PG, Lutzke L, et al. Recurrence of esophageal intestinal metaplasia after endoscopic mucosal resection and radiofrequency ablation of Barrett's esophagus: results from a US Multicenter Consortium. Gastroenterology 2013;145:79-86 e1.

17. Cook Medical. $2015 \mathrm{Gl}$ endoscopy coding and reimbursement guide. Bloomington, IN: Cook Medical, 2015.

18. Bureau of Labor Statistics. Consumer price index. United States Department of Labor. Available from: https://www.bls.gov/cpi. Accessed June 10, 2018.

19. de Koning HJ, Meza R, Plevritis SK, et al. Benefits and harms of computed tomography lung cancer screening strategies: a comparative modeling study for the U.S. Preventive Services Task Force. Ann Intern Med 2014;160:311-320.

20. Ubel PA, Hirth RA, Chernew ME, et al. What is the price of life and why doesn't it increase at the rate of inflation? Arch Intern Med 2003;163:1637-1641.

21. Gordon LG, Mayne GC, Hirst NG, et al. Cost-effectiveness of endoscopic surveillance of non-dysplastic Barrett's esophagus. Gastrointest Endosc 2014;79:242-256 e6.

22. Kastelein F, van Olphen S, Steyerberg EW, et al. Surveillance in patients with long-segment Barrett's oesophagus: a costeffectiveness analysis. Gut 2015;64:864-871.

23. Runge TM, Abrams JA, Shaheen NJ. Epidemiology of Barrett's esophagus and esophageal adenocarcinoma. Gastroenterol Clin North Am 2015;44:203-231.
24. Rubenstein JH, Mattek N, Eisen G. Age- and sex-specific yield of Barrett's esophagus by endoscopy indication. Gastrointest Endosc 2010;71:21-27.

25. US Preventive Services Task Force. Screening for colorectal cancer: US Preventive Services Task Force Recommendation Statement. JAMA 2016;316:545.

26. Siu AL, Bibbins-Domingo K, Grossman DC, et al. Screening for breast cancer: US Preventive Services Task Force Recommendation Statement. Ann Intern Med 2016;164:279.

27. NCl. Surveillance, Epidemiology, and End Results (SEER) program population (up to 2013). National Cancer Institute, DCCPS, Surveillance Research Program, Surveillance Systems Branch, Bethesda, MD, 2015.

28. de Jonge PJ, van Blankenstein M, Looman CW, et al. Risk of malignant progression in patients with Barrett's oesophagus: a Dutch nationwide cohort study. Gut 2010;59:1030-1036.

29. Hvid-Jensen F, Pedersen L, Drewes AM, et al. Incidence of adenocarcinoma among patients with Barrett's esophagus. N Engl J Med 2011;365:1375-1383.

30. Bhat S, Coleman HG, Yousef F, et al. Risk of malignant progression in Barrett's esophagus patients: results from a large population-based study. J Natl Cancer Inst 2011;103:1049-1057.

31. Rustgi AK, El-Serag HB. Esophageal carcinoma. N Engl J Med 2014;371:2499-2509.

32. Arnold M, Soerjomataram I, Ferlay J, et al. Global incidence of oesophageal cancer by histological subtype in 2012. Gut 2015; 64:381-387.

\section{Reprint requests}

Address requests for reprints to: Amir-Houshang Omidvari, MD, MPH, PO Box 2040, 3000 CA Rotterdam, The Netherlands. e-mail: a.omidvari@erasmusmc. nl; fax: (31) 10-703-84-74.

\section{CRediT Authorship Contributions}

Amir-Houshang Omidvari, MD, MPH (Conceptualization: Equal; Data curation: Equal; Formal analysis: Equal; Project administration: Equal; Validation: Equal; Visualization: Equal; Writing - original draft: Lead); Ayman Ali, BS (Data curation: Equal; Formal analysis: Equal; Writing - review \& editing: Equal); William D Hazelton, PhD (Conceptualization: Equal; Data curation: Equal; Formal analysis: Equal; Writing - review \& editing: Equal); Sonja Kroep, PhD (Conceptualization: Equal; Formal analysis: Supporting; Methodology: Equal; Writing review \& editing: Equal); Minyi Lee, BS (Data curation: Equal; Formal analysis: Equal; Visualization: Equal; Writing - review \& editing: Equal); Steffie K. Naber, PhD (Formal analysis: Supporting; Supervision: Supporting; Writing - review \& editing: Equal); Brianna N. Lauren (Formal analysis: Equal; Project administration: Equal; Validation: Equal; Writing - review \& editing: Equal); Sassan Ostvar, PhD (Formal analysis: Supporting; Writing - review \& editing: Equal); Ellen Richmond, RN, MS, AOCN (Conceptualization: Equal; Formal analysis: Supporting; Writing - review \& editing: Equal); Chun Yin Kong, PhD (Conceptualization: Equal; Formal analysis: Supporting; Writing - review \& editing: Equal); Joel H. Rubenstein, MD, MSC (Conceptualization: Equal; Data curation: Equal; Formal analysis: Supporting; Writing - review \& editing: Equal); Iris Lansdorp-Vogelaar, PhD (Conceptualization: Equal; Formal analysis: Supporting; Funding acquisition: Equal; Methodology: Equal; Resources: Equal; Supervision: Equal; Writing - original draft: Supporting; Writing - review \& editing: Equal); Georg Luebeck, PhD (Conceptualization: Equal; Formal analysis: Supporting; Funding acquisition: Equal; Resources: Equal; Supervision: Equal; Writing - review \& editing: Equal); Chin Hur, MD, MPH (Conceptualization: Equal; Data curation: Supporting; Formal analysis: Supporting; Funding acquisition: Equal; Resources: Equal; Supervision: Equal; Writing - review \& editing: Equal); John Inadomi, MD (Conceptualization: Equal; Data curation: Supporting; Formal analysis: Supporting; Funding acquisition: Equal; Resources: Equal; Supervision: Equal; Writing - original draft: Equal; Writing review \& editing: Equal).

\section{Conflicts of interest}

The authors disclose no conflicts.

\section{Funding}

All authors were supported by the National Institutes of Health/National Cancer Institute (U01CA152926 and U01CA199336). 